\title{
Avaliação dos parâmetros de Archie através de simulações numéricas em modelos digitais de rochas
}

\author{
Nathália Amorim Lima de Moraes ${ }^{1}$ \& José Agnelo Soares ${ }^{1},{ }^{1}$ PPGEPM - Universidade Federal de Campina Grande
}

\begin{abstract}
Copyright 2021, SBGf - Sociedade Brasileira de Geofísica
This paper was prepared for presentation during the $17^{\text {th }}$ International Congress of the Brazilian Geophysical Society held in Rio de Janeiro, Brazil, 16-19 August 2021.

Contents of this paper were reviewed by the Technical Committee of the $17^{\text {th }}$ International Congress of the Brazilian Geophysical Society and do not necessarily represent any position of the SBGf, its officers or members. Electronic reproduction or storage of any part of this paper for commercial purposes without the written consent of the Brazilian Geophysical Society is prohibited.
\end{abstract}

\section{Resumo}

A equação de Archie é o mais importante modelo utilizado para o cálculo das saturações fluidas em reservatórios de hidrocarbonetos. Essas saturações afetam diretamente na avaliação de reservas in situ, mas tal modelo depende de parâmetros de difícil mensuração. Neste trabalho é proposto um método, baseado na simulação numérica da propagação do campo elétrico sobre modelos digitais de rochas carbonáticas e areníticas, para a calibração dos parâmetros da equação de Archie. Imagens de microtomografia de raios- $x$ das amostras foram segmentadas em quatro domínios: poros internos, poros externos, fase intermediária e matriz. A análise dos modelos digitais permitiu quantificar propriedades topológicas das rochas como o fator de tortuosidade dos macroporos e a conectividade, através do número de Euler, dos quatro domínios que compõem os modelos digitais de rochas. Modelos tridimensionais das rochas foram gerados e representados por malhas numéricas sobre as quais foram simulados quatro cenários alternativos de saturação fluida. Para cada cenário foram determinados os parâmetros calibrados da equação de Archie e analisados os fatores que afetam esses parâmetros. A conectividade dos domínios condutivos controla os valores desses parâmetros.

\section{Introdução}

Archie (1942) publicou o mais importante artigo para a interpretação quantitativa de perfis geofísicos de poços, especialmente para a avaliação de reservas de óleo e gás. Naquele artigo clássico o autor apresenta duas equações. A primeira define o chamado Fator de Formação $(F)$, o qual relaciona a resistividade de uma rocha totalmente saturada com a resistividade da salmoura que a satura, além de uma relação entre $F$ e a porosidade da rocha estabelecendo o expoente $m$ como a inclinação da curva entre essas propriedades. A segunda equação estabelece a relação entre $\mathrm{F}$ e a saturação fluida, com a introdução do expoente $n$ como o seu fator de proporcionalidade. Mais tarde, Winsauer et al. (1952) propuseram uma modificação no modelo de Archie através da introdução do fator de tortuosidade (a). A forma atualmente mais conhecida da equação de Archie é dada por:

$S_{w}{ }^{n}=\frac{a R_{w}}{\emptyset^{m} R_{t}}$ onde $S_{w}$ é a saturação de água e $R_{w}$ é a resistividade da água que satura a rocha. $\varnothing$ é a porosidade da rocha e $R_{t}$ é a resistividade da rocha contendo hidrocarbonetos. O coeficiente a e os expoentes $m$ e $n$ são os parâmetros da equação de Archie. $m$ é conhecido como o expoente de cimentação e $n$ como o expoente de saturação.

O expoente de cimentação $m$ e o expoente de saturação $n$ são fontes de incerteza no cálculo da saturação fluida em reservatórios carbonáticos heterogêneos (Mardi et al., 2012). O expoente de cimentação seria igual a um se os poros de uma rocha fossem tubos cilíndricos retos e paralelos na direção da corrente elétrica (Olsen et al., 2008; Glover, 2010). No entanto, uma rocha sedimentar contém grãos de formato irregular, o que reduz a área da seção transversal efetiva do condutor, resultando em um fator de cimentação maior que a unidade. Aqueles autores afirmam que existe uma relação entre o expoente de cimentação e a superfície específica, a qual consiste em uma medida do tamanho de grãos ou de poros.

Dashtian et al. (2015) afirmam que $m$ aumenta à medida que a interconectividade dos poros diminui, além de indicar que a presença de argilominerais leva a uma estimativa menor e irreal do valor de $\mathrm{m}$. Liu et al. (2015), estudando tight gas sandstones, associam os valores dos parâmetros $m$ e $n$ de Archie à eficiência na condução da corrente elétrica através do sistema de poros saturados com fluidos condutivos. Para aqueles autores, quanto mais os poros forem bem interconectados maior será a eficiência elétrica da rocha e menor será o valor de $n$.

O valor de $n$ depende da saturação de água. Além da saturação, a forma como essa água condutiva se distribui no espaço poroso da rocha influencia fortemente na resistividade da rocha e, consequentemente, no valor de $n$ (Hamada, 2010). Para um amplo intervalo de saturações o valor do expoente de saturação diminui com o aumento de $S_{w}$.

Sen (1997) afirma que em carbonatos microporosos $n$ diminui com o aumento de participação da microporosidade. Se esses microporos tornam-se eletricamente desconectados $n$ aumenta. Tsakiroglou \& Fleury (1999) indicam que a variação do expoente de saturação em função da saturação de água enfraquece em meios com alta conectividade da rede de poros. Cerepi (2004) aponta que a condutividade elétrica não depende apenas da porosidade, mas também é fortemente sensível à microestrutura do sistema poroso, à conectividade do espaço poroso e à sua microgeometria. Em rochas carbonáticas os microporos fornecem a conectividade e 0 caminho do fluido entre escalas maiores de poros, de outra forma desconectadas (Yao et al., 2013; Harland et al., 2015). A conectividade da rede de poros pode ser estimada pelo número de Euler (Harland et al., 2015). 
Neste artigo os parâmetros da equação de Archie são determinados para seis amostras digitais de rocha, sendo três carbonáticas e três areníticas. Tais parâmetros são calculados através da análise topológica dos modelos digitais das rochas e da simulação numérica, pelo método de elementos finitos, da propagação de um campo elétrico para diferentes cenários de saturação fluida. Os modelos digitais de rocha foram obtidos por microtomografia de raios $\mathrm{x}$.

\section{Método}

Neste trabalho são analisadas seis amostras de rochas provenientes de formações geológicas de bacias sedimentares dos EUA. São três amostras de rochas carbonáticas e três de rochas areníticas.

A amostra AC_12 é um carbonato matriz-suportado de granulometria fina composto predominantemente por microorganismos. É composta principalmente por calcita e secundariamente por componentes siliciclásticos como argilas, quartzo e albita (Loucks et al., 2021). A amostra IL3_20 é um calcarenito de granulometria grossa a média, poroso e medianamente bem selecionado. Os grãos individuais são majoritariamente microfósseis e fragmentos de macrofósseis. Comumente ocorre textura oolítica com grãos revestidos e sua composição mineral é predominantemente calcítica (Brisbin et al., 2005). Por sua vez a amostra SD_12 é composta quase que exclusivamente por dolomita, com cristais bem desenvolvidos, predominantemente de tamanho areia e porosidade do tipo intergranular.

A amostra PSS_02 consiste em um arenito com grãos de tamanho areia média a fina, composta majoritariamente por grãos de quartzo e com porosidade predominante do tipo intergranular. A amostra CGS_15 é um arenito de origem fluvial composto principalmente de quartzo, sendo pobremente cimentado com calcita e contém uma pequena percentagem de argila. $\mathrm{O}$ tamanho médio de grãos é $0,2 \mathrm{~mm}$ (Ingraham et al., 2013). A amostra SCS 01 é um arenito fino a muito fino, com considerável participação de grãos de quartzo e argilominerais.

Das imagens digitais de cada amostra de rocha foi extraído, do centro da amostra digital, um cubo de dados com $200^{3}$ pixels. Esses cubos de dados foram utilizados para todas as análises subsequentes. O tamanho de pixel das imagens variou entre 2,17 $\mu \mathrm{m}$ e 2,65 $\mu \mathrm{m}$. Cada cubo de dados foi segmentado em quatro domínios: 1) poros internos (corpo dos poros); 2) poros externos (região dos poros adjacente aos grãos, aquela que contém água irredutível ou óleo residual); 3) fase intermediária (região microporosa); e 4) matriz mineral.

Em seguida foi realizada a análise da conectividade dos domínios potencialmente condutivos. A matriz mineral é considerada infinitamente resistiva em todos os cenários de saturação simulados numericamente, de modo que a investigação topológica somente é realizada para os demais domínios, os quais podem conter fluidos condutivos. Adota-se neste trabalho, como um indicador de conectividade, um índice determinado a partir do número de Euler obtido, pela análise do modelo digital de rocha, para cada domínio potencialmente condutivo. $\mathrm{O}$ número de Euler é um número grande e negativo em um domínio fortemente interconectado, e um número grande positivo em um domínio com baixa interconectividade. A fim de obter um índice com o mesmo sinal do número de Euler (NE), mas com valores contidos em um intervalo menor de valores, neste trabalho o índice indicador de conectividade (IC) foi definido como o logaritmo decimal do valor absoluto do número de Euler. Após a aplicação da função logaritmica dá-se ao índice o mesmo sinal do númer de Euler original.

Foram geradas malhas tridimensionais de elementos tetragonais representativas dos modelos digitais das amostras de rocha. Cada malha contém as propriedades de volume, forma e topologia dos quatro domínios e se destina à simulação numérica das propriedades elétricas sob diferentes cenários de saturação fluida.

Para a simulação numérica foram adotados quatro cenários alternativos de saturação fluida: 1) A rocha está $100 \%$ saturada com água, ou seja, os macroporos (poros internos e externos) são condutivos; 2) A rocha contém água nos poros externos e óleo nos poros internos; 3) A rocha contém óleo nos poros externos, mas contém água nos poros internos; 4) A rocha contém óleo tanto nos poros externos como nos poros internos. Em todos os quatro cenários os microporos contidos na fase intermediária são condutivos. Foi aplicada uma diferença de potencial elétrico de 12 volts entre as extremidades inferior e superior de cada malha numérica, cujo resultado fornece a distribuição espacial do potencial elétrico e a densidade de corrente $(\mathcal{l}$ ) para cada cenário de saturação investigado.

O fator de tortuosidade foi estimado diretamente dos modelos digitais de rocha, através da aplicação da função centroid path tortuosity do software Avizo Fire ${ }^{\circledR}$. Os expoentes de cimentação e de saturação foram determinados através da manipulação das equações do Fator de Formação e de Archie, respectivamente. Para tanto essas equações foram linearizadas pela aplicação da função algorítmica.

Considerando que a simulação numérica fornece o valor da densidade de corrente, a resistividade da rocha para cada cenário de saturação fluida é dada pela razão entre a tensão aplicada e o produto entre a densidade de corrente e o comprimento da amostra.

Análises estatísticas foram aplicadas aos dados a fim de avaliar a relação entre os parâmetros da equação de Archie, determinados a partir das propriedades elétricas simuladas numericamente, e os índices de conectividade dos domínios potencialmente condutivos dos modelos digitais de rocha.

\section{Resultados}

As malhas numéricas construídas a partir das imagens microtomográficas são apresentadas na Figura 1. Os poros internos são formados essencialmente por poros grandes, os poros externos são poros de tamanho médio a pequeno, e a fase intermediária consiste no domínio microporoso que, nas amostras carbonáticas corresponde à matriz micrítica, e nas amostras arenosas corresponde aos argilominerais. Os poros externos podem conter água irremovível ou óleo residual, a depender do cenário de saturação. 

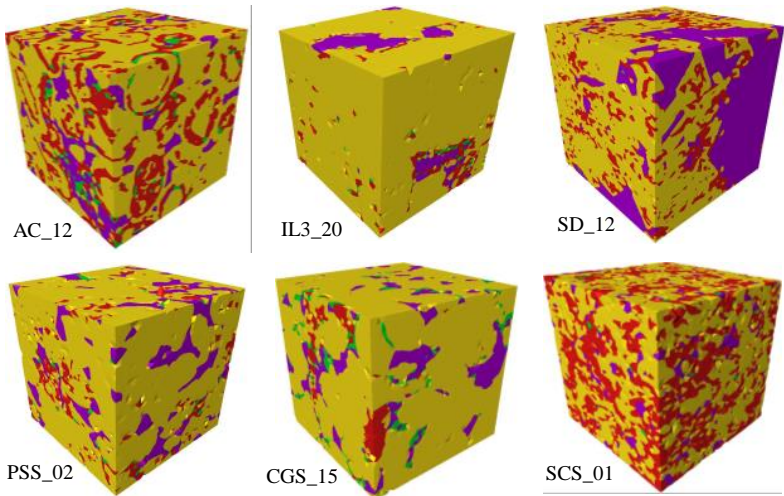

Figura 1 - Malhas numéricas geradas. As cores indicam os domínios: amarelo $\rightarrow$ matriz mineral, roxo $\rightarrow$ poros internos, verde $\rightarrow$ poros externos, e vermelho $\rightarrow$ fase intermediária microporosa.

A Figura 2 mostra que na maioria das amostras os macroporos internos predominam nas malhas numéricas, com exceção das amostras AC_12 e SCS_01, onde os microporos são volumetricamente mais importantes.

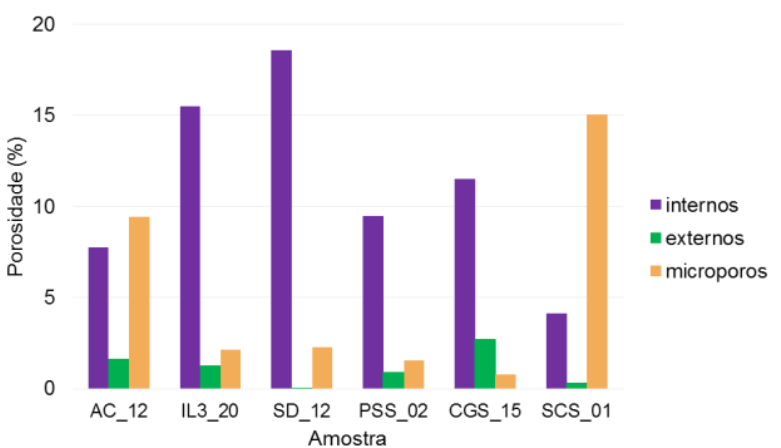

Figura 2 - Porosidade dos poros internos, externos e dos microporos.

A Figura 3 apresenta o índice de conectividade (IC) dos domínios potencialmente condutivos de todas as amostras. Valores de IC fortemente positivos indicam baixa conectividade e valores fortemente negativos são associados à elevada conectividade. Observa-se que os poros externos (PE) são essencialmente mal conectados em quase todas as amostras. Uma exceção é a amostra SD_12. No entanto, essa é uma amostra onde o volume de poros externos é desprezível. Os poros internos (PI) são mais interconectados que os poros externos, com exceção da amostra SCS_01, a qual possui a menor presença de poros internos dentre todas as amostras. Já a fase intermediária $(\mathrm{FI})$ microporosa é muito bem interconectada nas amostras AC 12, SD 12 e SCS 01. Essa característica juntamente com a elevada presença de microporos nas amostras AC_12 e SCS_01 tornam a fase intermediária dominante sobre as propriedades elétricas dessas duas amostras.

As figuras 4 a 7 apresentam as distribuições espaciais do potencial elétrico simulado para os quatro cenários de saturação fluida nas amostras AC_12, IL3-20, CGS_15 e SCS_01. No cenário (A) água satura tanto os poros internos como os externos. No cenário (B) há óleo nos poros internos e água irremovível nos poros externos. No cenário $(C)$ água preenche os poros internos e nos poros externos há óleo residual; e no cenário (D) tanto os poros internos como os externos contêm óleo. Em todos os cenários a fase intermediária microporosa é considerada condutiva pois está saturada com água da formação.

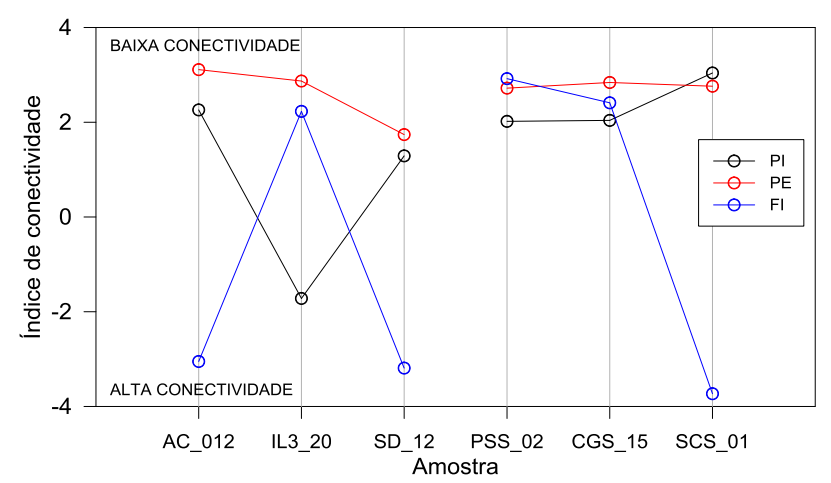

Figura 3 - Índice de conectividade dos domínios potencialmente condutivos.

A amostra AC-12 é mais microporosa e possui menor volume de poros internos do que a amostra IL3 20. Além disso, os poros internos da amostra AC_12 são menos interconectados do que os da amostra IL3_20. Por sua vez a fase intermediária microporosa da amostra AC-12 é mais interconectada do que a da amostra IL3_20. Os poros externos de ambas as amostras são mal conectados. A mudança no padrão de distribuição do potencial elétrico devido à variação na condutividade do fluido contido nos poros internos é aparentemente mais forte na amostra AC_12 do que na amostra IL3_20. Isto sugere que nessas amostras carbonáticas ocorre uma interação elétrica entre os fluidos contidos nos macroporos internos com os fluidos contidos nos microporos, por essa razão o efeito da mudança de fluido contido nos poros internos da amostra AC 12 é maior do que na amostra IL3 20, pois a conectividade adicional oferecida pela rede de microporos da AC-12 supera o efeito da rede de macroporos internos da IL3_20. O efeito causado pela mudança do fluido contido nos poros externos é pequeno em ambas as amostras.

Em relação às amostras siliciclásticas se observa que a amostra SCS_01 possui uma fase intermediária microporosa muito maior e mais interconectada do que a amostra CGS 15. Por outro lado, o volume de poros internos da amostra SCS_01 é bem menor do que o da amostra CGS 15. A porosidade proporcionada pelos poros externos é consideravelmente menor na amostra SCS_01 do que na amostra CGS_15, embora esses poros sejam mal interconectados em ambas as amostras. Diferentemente das amostras carbonáticas anteriormente discutidas, a amostra arenosa de granulometria mais fina, mais rica em microporos e com menor participação de macroporos internos (SCS 01) apresenta um padrão de distribuição espacial do potencial elétrico menos afetado pela mudança da condutividade do fluido contido nos macroporos internos. Isto ocorre porque o volume de poros internos da amostra SCS 01 é bem menor do que o da amostra CGS 15, de forma que a distribuição do potencial elétrico é controlado pela fase intermediária microporosa. Já no caso da amostra CGS_15 a microporosidade não interfere, pois ela é pequena e mal interconectada, de modo que o padrão espacial do 
potencial elétrico é principalmente afetado pela condutividade do fluido contido nos poros internos e secundariamente afetado pelo fluido que satura os poros externos.
(A)

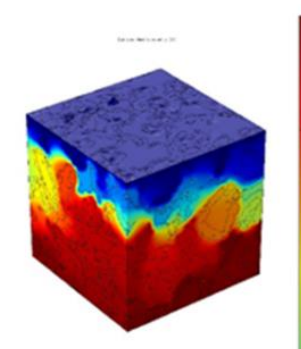

(C)

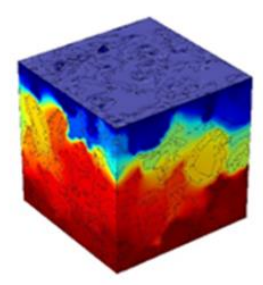

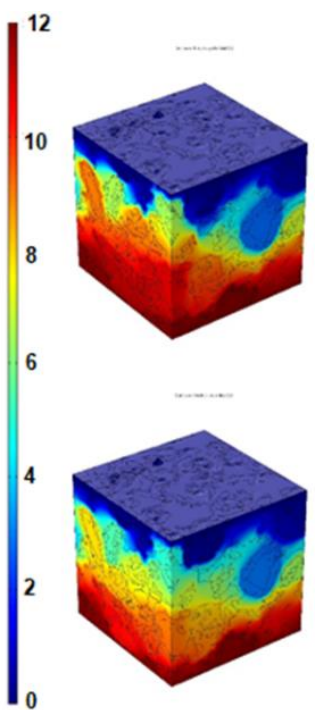

(B)

(D)
(A)

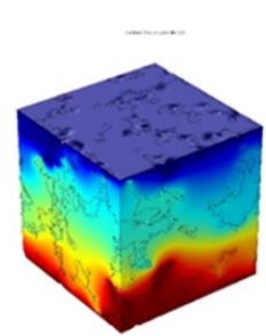

(C)

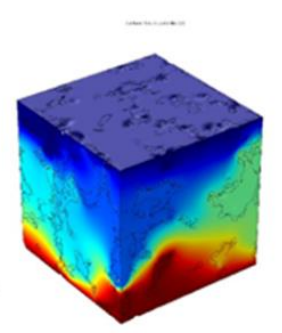

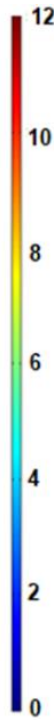

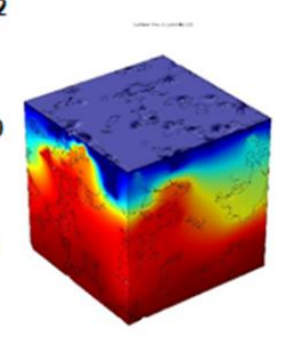

6

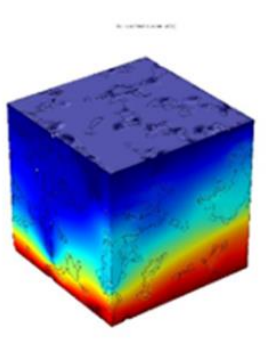

(B)

Figura 6 - Distribuição do potencial elétrico na amostra CGS_15 para os quatro cenários de saturação fluida. Escala de cores em Volts.
(A)

AC_12 para os quatro cenários de saturação fluida. Escala de cores em Volts.

(A)

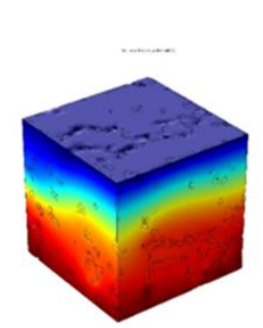

(C)

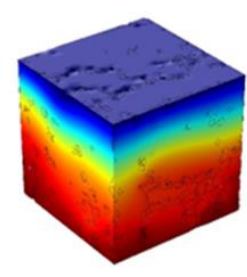

(B)

(D)

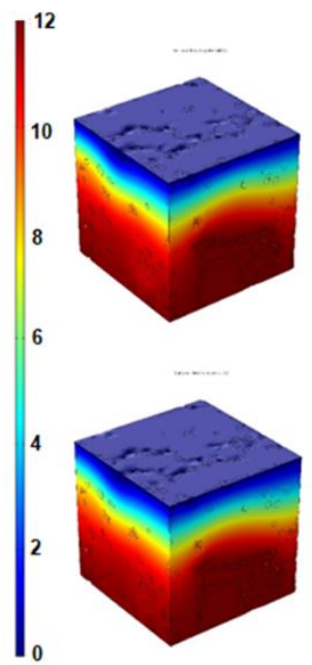

Figura 5 - Distribuição do potencial elétrico na amostra IL3_20 para os quatro cenários de saturação fluida. Escala de cores em Volts.

Os valores do fator de tortuosidade estimados são apresentados na Figura 8 . Todos os valores se encontram no intervalo entre 1,71 e 2,29. Há uma relação inversa entre o fator de tortuosidade e a porosidade dos macroporos. Por exemplo, as amostras IL3_20 e SD-12, que exibem maior porosidade dos macroporos (poros internos mais poros externos), são as que apresentam menores valores para o fator de tortuosidade.

Na Figura 9 são apresentados os valores do expoente de cimentação. Nela vê-se que as duas amostras (AC_12 e SCS 01) nas quais predomina a microporosidade os valores para o expoente de cimentação são consideravelmente maiores que nas demais amostras.

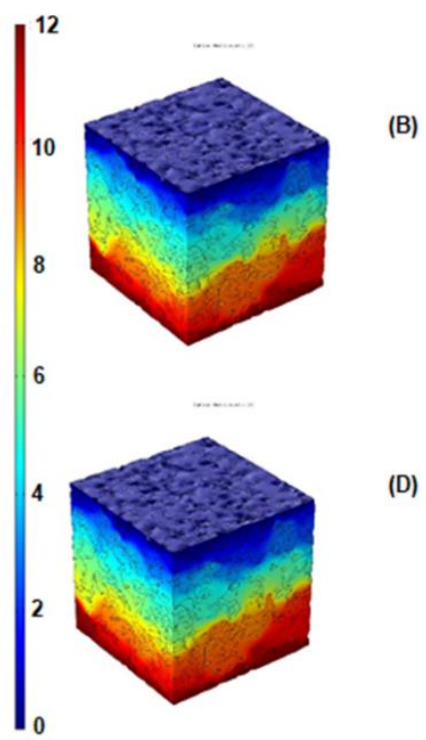

Figura 7 - Distribuição do potencial elétrico na amostra SCS_01 para os quatro cenários de saturação fluida. Escala de cores em Volts.

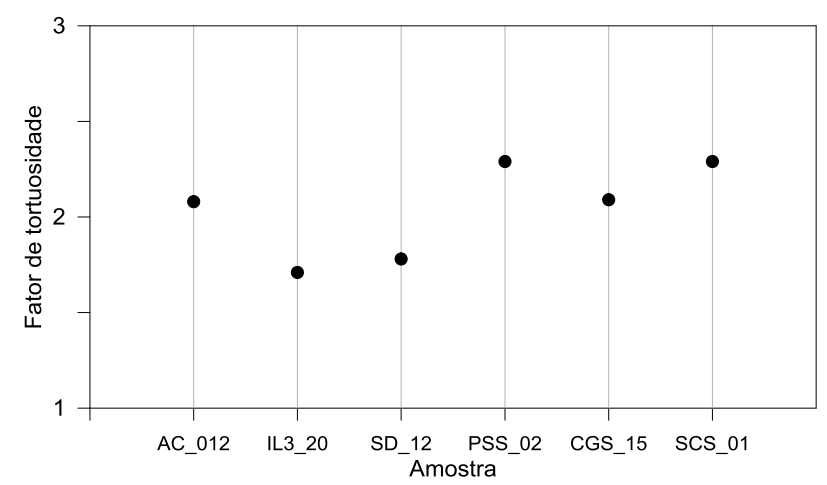

Figura 8 - Valores estimados para o fator de tortuosidade. 


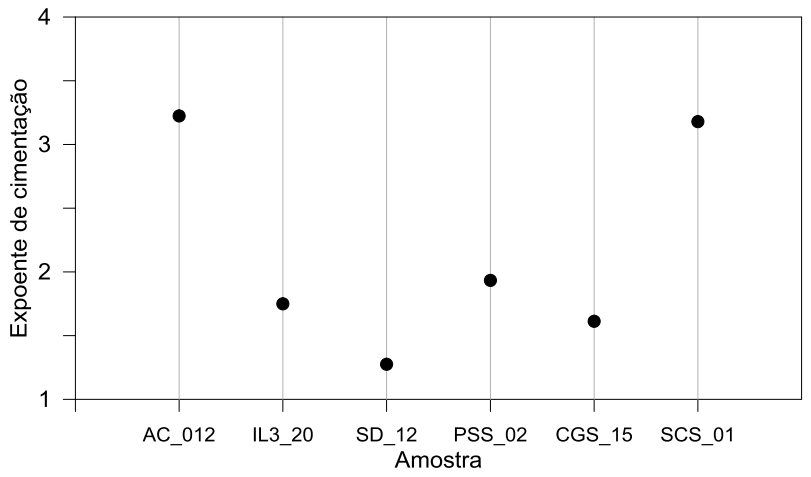

Figura 9 - Valores estimados para o expoente de cimentação.

A Figura 10 apresenta os valores dos expoente de saturação para os cenários de saturação 2,3 e 4 . Nela vêse que todas as amostras quando possuem óleo saturando os seus poros internos apresentam valores de $n$ no intervalo entre 2 e 4 , de modo quase independente do fluido contido nos seus poros externos. Por outro lado, quando os poros internos estão totalmente saturados com água, embora exista óleo residual em seus poros externos, os valores de $n$ variam de forma diferente entre amostras carbonáticas e siliciclásticas. Nas amostras carbonáticas o valor de $n$ diminui em relação aos cenários em que os poros internos contêm óleo, enquanto que nas amostras arenosas o valor de $n$ aumenta. Isto parece resultar da interação elétrica entre os fluidos contidos nos macroporos internos e nos microporos da fase intermediária. Nas amostras carbonáticas, de acordo com a Figura 3, ao menos um desses domínios apresenta alto IC, permitindo trocas elétricas entre os fluidos. Já nas amostras arenosas apenas a SCS_01 apresenta um desses domínios (FI) bem interconectado, no entanto também apresenta um baixo volume de poros internos muito mal conectados. Estes resultados indicam a importância da interação entre os fluidos contidos em poros com diferentes tamanhos e propriedades topológicas para a determinação dos expoentes da equação de Archie.

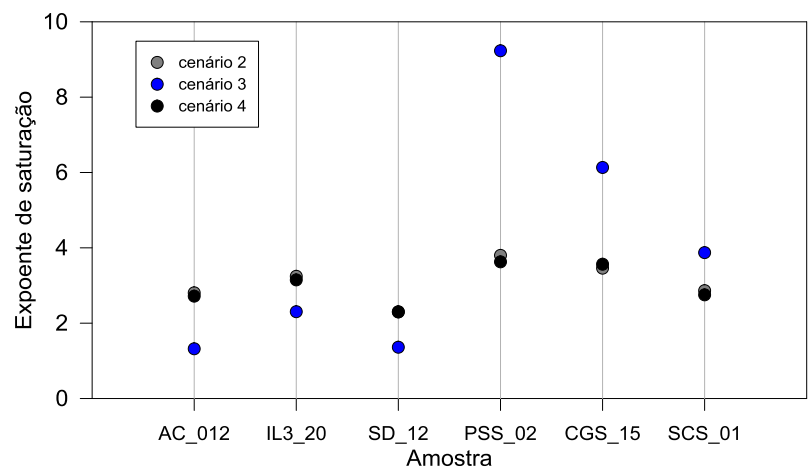

Figura 10 - Valores do expoente de saturação estimados pela simulação numérica para os cenários de saturação 2 , 3 e 4.

Aplicando a análise de regressão linear piecewise foram obtidos modelos preditivos para os parâmetros da equação de Archie considerando apenas os índices de conectividade dos domínios potencialmente condutivos como variáveis independentes. A regressão piecewise fornece duas equações preditivas: uma válida para valores da variável a estimar abaixo do breakpoint e outra para valores acima do breakpoint.

Para a predição do fator de tortuosidade o valor do breakpoint foi $a=2,04$ e as equações de predição foram:

$a=0,408-1,059 I C(P I)+0,341 I C(P E)-0,672 I C(F I)$ para $a \leq 2,04 \mathrm{e}$

$a=10,007-0,792 I C(P I)-2,105 I C(P E)-0,134 I C(F I)$ para $a \geq 2,04$.

Para a predição do expoente de cimentação o valor do breakpoint foi $m=2,162 \mathrm{com}$ as seguintes equações de predição:

$m=4,635-0,062 I C(P I)-1,293 I C(P E)+0,323 I C(F I)$

para $m \leq 2,162 \mathrm{e}$

$m=0,258+0,004 I C(P I)+0,678 I C(P E)-0,278 I C(F I)$ para $m \geq 2,162$.

Já para a predição dos valores do expoente de saturação, para cada cenário de saturação fluida há duas equações preditivas. As equações para o cenário 2 (reservatório com óleo nos poros internos e com água irremovível nos poros externos) são:

$n=0,332-0,067 I C(P I)+0,456 I C(P E)-0,396 I C(F I)$

para $n \leq 3,08$ e

$n=0,254+0,024 I C(P I)+0,455 I C(P E)+0,773 I C(F I)$

para $n \geq 3,08$.

O coeficiente de determinação para todas as funções de regressão piecewise foram iguais a 1, ou seja, ocorreu uma predição perfeita dos parâmetros da equação de Archie a partir dos índices de conectividade dos domínios potencialmente condutivos. Embora este resultado precise ser confirmado em um número muito maior de amostras, ele sugere uma solução relativamente simples para a calibração desses parâmetros em intervalos específicos dos reservatórios. Tal calibração de parâmetros conduz a uma estimativa de saturação fluida mais precisa e, por consequência, menor incerteza na avaliação de uma dada formação geológica.

\section{Discussão}

Os valores observados para o fator de tortuosidade foram todos levemente diferentes de 2 , portanto maiores que 0 valor unitário previsto originalmente por Archie (1942) e mais compatíveis com o modelo modificado por Winsauer et al. (1952). Há uma relação inversa entre o fator de tortuosidade e a porosidade dos macroporos.

Os valores observados para o expoente de cimentação são maiores que 1 e apresentam uma clara relação com o tamanho dos grãos minerais e, portanto, dos poros. As amostras de grãos mais finos foram as que apresentaram maiores valores de $m$. Isto é compatível com as afirmações de Olsen et al. (2008) e de Glover (2010). Além disso, os valores de $m$ apresentam uma inequívoca relação com a conectividade dos domínios potencialmente condutivos (poros internos, poros externos e fase intermediária microporosa), conforme discutido por Dashtian et al. (2015) e Liu et al. (2015). Estes últimos autores afirmam que quanto mais os poros forem bem interconectados maior será a eficiência elétrica da rocha e 
menor será o valor de $n$. Esse comportamento fica bem evidenciado quando se comparam as figuras 3 e 9 .

Sen (1997) afirma que em carbonatos microporosos se os microporos tornam-se eletricamente desconectados $n$ aumenta. Esse comportamento é confirmado pelas análises realizadas neste trabalho. Para perceber tal relação basta comparar os valores do índice de conectividade da fase intermediária (FI) microporosa, da Figura 3, com os valores de $n$ apresentados na Figura 10.

A importância da interação entre os fluidos contidos em poros com diferentes tamanhos e propriedades topológicas para a determinação dos expoentes da equação de Archie, sugerida pelos resultados desta pesquisa, confirma a discussão apresentada por Yao et al. (2013) e Harland et al. (2015).

\section{Conclusões}

A simulação numérica da propagação do campo elétrico em modelos digitais de rocha associada à análise topológica de seus domínios permitiu estimar os valores do parâmetros da equação de Archie para tipos específicos de rochas-reservatório. Isto se constitui em um novo método para a determinação dos parâmetros de Archie.

O padrão de resistividade, observado para os quatro cenários de saturação fluida simulados, indica que a resistividade elétrica da rocha é dominada pelo grau de conectividade dos domínios condutivos. O método apresentado neste trabalho pode ser aplicado para simular outros diferentes cenários de saturação fluida.

A porosidade e a conectividade dos poros internos exerce grande influência sobre o fator de tortuosidade, enquanto o expoente de cimentação é controlado pelo índice de conectividade da fase intermediária e dos poros externos. Já o expoente de saturação é controlado pela conectividade da fase intermediária em todos os casos investigados e adicionalmente pela conectividade dos poros internos quando os poros internos contém água e os poros externos contém óleo.

Modelos estatísticos permitiram estimar os parâmetros da equação de Archie, para os diferentes cenários de saturação fluida, a partir da conectividade (número de Euler) dos domínios porosos. Isso implica que, se os modelos estatísticos forem robustos, a metodologia aqui desenvolvida simplifica muito o processo de determinação dos parâmetros da equação de Archie.

A calibração dos parâmetros da equação de Archie para rochas-reservatório específicas tem potencial para exercer um considerável impacto sobre a atividade de avaliação de formações, resultando em quantificações de reservas mais confiáveis e influenciando na análise da economicidade de um dado prospecto.

\section{Agradecimentos}

Os autores agradecem a PETROBRAS pelo fornecimento e imageamento das amostras de rocha através de vários termos de cooperação que se utilizam dos recursos das participações especiais junto à ANP. Nathália Moraes agradece à CAPES pela bolsa de pesquisa condedida.

\section{Referências}

Archie, G.E., 1942. The electrical resistivity log as an aid in determining some reservoir characteristics: T. AIME, 146, 54-62.

Brisbin, W.C., Young, G. and J. Young, 2005. Geology of the Parliament Buildings 5: Geology of the Manitoba Legislative Building. Geoscience Canada, Vol. 32, N. 4.

Cerepi, A., 2004. Geological control of electrical behaviour and prediction key of transport properties in sedimentary porous systems: Colloids and Surfaces A: Physicochem. Eng. Aspects, 241, 281-298.

Glover, P.W.J., 2010. A generalized Archie's law for $n$ phases: Geophysics, 75, no. 6, E247-E265.

Hamada, G.M., 2010. Analysis of Archie's parameters determination techniques: Petroleum Science and Technology, 28:1, 79-92.

Harland, S.R., R.A. Wood, A. Curtis, M.I.J. van Dijke, K. Stratford, Z. Jiang, W. Kallel, and K. Sorbie, 2015. Quantifying flow in variably wet microporous carbonates using object-base geological modeling and both latticeBoltzmann and pore-network fluid flow simulations: AAPG Bulletin, v. 99, no. 10, 1827-1860.

Ingraham, M.D., Issen, K.A. and D.J. Holcomb, 2013. Response of Castlegate sandstone to true triaxial states of stress. Journal of Geophysical Research: Solid Earth, Vol. 118, 536-552.

Loucks, R.G., Reed, R.M., Ko, L.T., Zahm, C.K. and T.E. Larson, 2021. Micropetrographic characterization of a siliciclastic-rich chalk; Upper Cretaceous Austin Chalk Group along the onshore northern Gulf of Mexico, USA. Sedimentary Geology, 412, 105821.

Liu, H., Y. Zhao, Y. Luo, Z. Chen and S. He, 2015. Diagenetic facies controls on pore structure and rock electrical parameters in tight gas sandstone: J. Geophys. Eng. 12, 587-600.

Mardi, M., H. Nurozi and S. Edalatkhah, 2012. A water saturation prediction using artificial neural networks and an investigation on cementation factors and saturation exponent variations in an Iranian oil well: Petroleum Science and Technology, 30:4, 425-434.

Olsen, C., T. Hongdul, and I.L. Fabricius, 2008. Prediction of Archie's cementation factor from porosity and permeability through specific surface: Geophysics, Vol. 73, no. 2, E81-E87.

Sen, P.N., 1997. Resistivity of partially saturated carbonate rocks with microporosity: Geophysics, Vol. 62, no. 2, 415425.

Tsakiroglou, C.D., and M. Fleury, 1999. Pore network analysis of resistivity index for water-wet porous media: Transport in Porous Media, 35, 89-128.

Winsauer, W.O., Shearin Jr., H.M., Masson, P.H. and M. Williams, 1952, Resistivity of brine-saturated sands in relation to pore geometry: AAPG Bulletin, 36 (2): 253-277.

Yao, J., C. Wang, Y. Yang, R. Hu, and X. Wang, 2013. The construction of carbonate digital rock with hybrid superposition method: Journal of Petroleum Science and Engineering, 110, 263-267. 